Revista Brasileira de Meteorologia, v.22, n.1, 105-111, 2007

\title{
ANÁliSE COMPARATIVA DOS ÍNDICES DE SECA DE PALMER, PALMER ADAPTADO E ÍNDICE PADRONIZADO DE PRECIPITAÇÃO NO ESTADO DE SÃO PAULO
}

\author{
GABRIEL CONSTANTINO BLAIN ${ }^{1}$ e ORIVALDO BRUNINI ${ }^{2}$
}

\author{
1,2 Instituto Agronômico de Campinas (IAC). \\ Av. Barão de Itapura, 1487, CEP 13001-970, Caixa postal 28, Botafogo, Campinas, São Paulo. \\ E-mail: ${ }^{1}$ gabriel@iac.sp.gov.br, ${ }^{2}$ brunini@iac.sp.gov.br
}

Recebido Agosto 2005 - Aceito Julho 2006

\begin{abstract}
RESUMO
O Índice Padronizado de Precipitação (SPI) e o Índice de Severidade de Seca de Palmer, tanto na sua forma original (PDSI) quanto na sua forma adaptada às condições climáticas do Estado de São Paulo (PDSI adap) vêm sendo utilizados pelo Instituto Agronômico (IAC) nas implicações agrícolas, meteorológicas, hidrológicas e sócio-econômicas do fenômeno seca. Esse estudo teve como objetivo a análise comparativa entre o SPI, o PDSI e o PDSI adap, respeitando-se as características inerentes de cada um desses modelos. Esse trabalho fornece ainda informações aos usuários sobre o correto uso e interpretação dos resultados das ferramentas em questão. Devido à sua versatilidade, consistência espacial e a fácil interpretação de seus resultados, o SPI deve ser utilizado na análise dos riscos, mitigação e implicações do fenômeno seca nos diversos segmentos da sociedade. O método do PDSI pode ser utilizado no Estado de São Paulo em sua forma adaptada (PDSI adap) para a quantificação da seca meteorológica na escala mensal.
\end{abstract}

Palavras-chave: déficit de precipitação, análise dos riscos, mitigação.

\begin{abstract}
COMPARING THE STANDARDIZED PRECIPITATION INDEX, THE PALMER DROUGHT SEVERITY INDEX AND THE PALMER ADAPTED INDEX ON THE STATE OF SÃO PAULO- BRAZIL.

The Standardized Precipitation Index (SPI) and the Palmer Drought Severity Index, on it's original form (PDSI) and on it's climate conditions of the State of São Paulo adapted model (PDSI adap) are been used by the Instituto Agronômico (IAC) through the CIIAGRO to evaluate the agricultural, the meteorological, the hydrological and the socioeconomic impacts of a drought. Regarding the particular characteristics of each index, this study compares the SPI, the PDSI and the PDSI adap models. This work also gives information about the correct interpretations of the results for each drought index. Due it's capability to recognizes the importance of time scales in the analysis of water availability and water use, due it's spatial consistence and it's simplicity interpretation the SPI should be used on studies of drought risk and mitigation over different segments of the human society. The PDSI adap could be used as a "meteorological drought monitoring tool", in monthly basis, on the State of São Paulo.
\end{abstract}

Keywords: precipitation deficit, drought risk and mitigation.

\section{INTRODUÇÃO}

Atualmente, o monitoramento do fenômeno seca utiliza índices que padronizam em base espacial e temporal essa anomalia climática. O Índice de Severidade de Seca de Palmer (PDSI) e o Índice Padronizado de Precipitação (SPI) são os mais conhecidos e mundialmente utilizados. A adaptação do método do PDSI às condições hídricas do Estado de São Paulo, realizadas por Blain (2005) resultou em um novo índice denominado PDSI adap. Esses três índices vêm sendo utilizados pelo Centro
Integrado de Informações Agrometeorológicas (CIIAGRO) do Instituto Agronômico (IAC) no estudo e análise das implicações agrícolas, meteorológicas, hidrológicas e sócio-econômicas do fenômeno seca.

De acordo com Guttman (1998) o PDSI é um quantificador da severidade de uma seca, sendo que um único valor do índice é usado para suspender ou implantar planos de contingência dessa anomalia climática. $\mathrm{O}$ índice deve ser calculado com base em uma série histórica de pelo menos trinta anos de dados de temperatura do ar e de precipitação 
pluvial, na escala mensal. É interessante ainda ressaltar que Palmer (1965) desenvolveu o PDSI como um indicador da seca meteorológica sem, entretanto, especificar qual escala de tempo (memória) o índice considera em sua análise. Essa indefinição temporal, o uso de regras arbitrárias para definir o início ou o fim de uma seca e a demora (exagerada memória) em responder à mudanças no regime de precipitação são características inerentes ao PDSI que são criticadas por Aley (1984), Karl (1986), Mckee et al., (1993) e Hayes et al., (1999).

Tais críticas serviram de base para o desenvolvimento do PDSI adap, o qual possui escala de tempo definida de análise (mensal), não apresenta a memória exagerada do método original e apresenta as definições do início ou do fim de uma seca relacionadas à razão entre a evapotranspiração real (ETR), calculada com base no método de Thornthwaite e Mather (1955) e a evapotranspiração potencial (ETP). Entretanto, assim como o PDSI, o PDSI adap utiliza apenas um único valor do índice, considerando com isso apenas as implicações de um tipo de seca - a meteorológica; a qual tem seu início detectado quando o PDSI adap "cai abaixo" de -1 e seu fim estabelecido quando a magnitude de -0,5 é alcançada.

Desenvolvido por Mckee et al., (1993) o SPI é a única ferramenta conhecida capaz de quantificar ou monitorar a seca em diferentes escalas de tempo. Para esses autores, tais escalas ao serem relacionadas ao déficit de precipitação, tornam-se extremamente importantes na caracterização de diferentes tipos de seca como, por exemplo, a meteorológica, a hidrológica e a sócio-econômica. $\mathrm{O}$ evento seca começa quando o SPI torna-se negativo e atinge o valor de -1 e termina quando este volta a apresentar valores positivos. Esse índice tem como base o ajuste de séries de precipitação à distribuição gama incompleta. Tal distribuição é adequada para descrever o comportamento de variáveis que possuam limite inferior superior a zero (THOM, 1966). Segundo Guttman (1998) o SPI, ao ser comparado ao PDSI, representa melhor os possíveis períodos de déficit ou excesso de precipitação.

A comparação entre o PDSI, o PDSI adap e o SPI deve ser feita observando-se as características inerentes de cada um desses índices. A tentativa de se estabelecer uma correlação entre o Índice de Severidade de Seca de Palmer, sem se conhecer qual escala de tempo essa ferramenta considera em sua análise, e o Índice Padronizado de Precipitação, pode levar o leitor a interpretações erradas sobre a análise do fenômeno seca.

Assim, esse estudo teve como objetivo a análise comparativa entre o SPI, o PDSI e o PDSI adap. Espera-se ainda com esse trabalho fornecer informações aos usuários sobre o correto uso e interpretação dos resultados dos índices em questão.

\section{MATERIAL E MÉTODOS}

Com base em dados da rede de Estações Meteorológicas do Instituto Agronômico da Secretaria de Agricultura e Abastecimento, foram gerados balanços hídricos mensais com período homogêneo, entre 1961 a 2000, para as localidades de Campinas

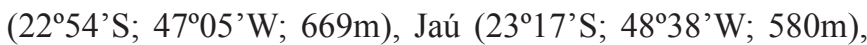
Mococa $\left(21^{\circ} 28^{\prime} \mathrm{S} ; 51^{\circ} 05^{\prime} \mathrm{W} ; 665 \mathrm{~m}\right)$, Piracicaba $\left(22^{\circ} 43^{\prime} \mathrm{S}\right.$; $\left.47^{\circ} 38^{\prime} \mathrm{W} ; 580 \mathrm{~m}\right)$, Ribeirão Preto (21 $\left.{ }^{\circ} 11^{\prime} \mathrm{S} ; 47^{\circ} 48^{\prime} \mathrm{W} ; 620 \mathrm{~m}\right)$, Tatuí (2322'S 4752’W; 600m).

O Índice Padronizado de Precipitação quantifica o déficit ou o excesso de precipitação em diferentes escalas de tempo. Tal característica torna o SPI uma valiosa ferramenta nos diversos estudos de disponibilidade hídrica; sejam eles de curta ou longa duração (Hayes et al, 1999). O cálculo do índice inicia-se com o ajuste da função densidade de probabilidade gama às séries de precipitação mensal. Após essa etapa a probabilidade acumulada de ocorrência de cada total mensal observado é estimada. A função normal inversa (Gaussiana) é aplicada a essa probabilidade. O resultado é o SPI.

Uma das principais etapas do modelo do PDSI é o calculo da precipitação "Climatologicamente Apropriada às Condições Existentes" (P) que pode ser entendida como o valor de precipitação mensal necessário para que uma determinada área permaneça sob condições climáticas normais. Tal parâmetro será calculado como descrito por Palmer (1965).

Para o cálculo da anomalia hídrica mensal (d), a precipitação observada no mês (Pi) é comparada a $\mathrm{P}$ do mesmo período:

$$
\mathrm{d}=\mathrm{Pi}-\mathbf{P}
$$

Como Palmer (1965) objetivava desenvolver um índice padronizado comparável em diferentes localidades e em qualquer época, d teve de ser padronizado (ponderado) em base regional (Karl, 1986). Assim, Palmer (1965) desenvolve um fator de caracterização climática designado pela letra K.

$$
\begin{aligned}
& \mathrm{K}=17,67 * \mathrm{~K}^{\prime} / \sum_{\mathrm{i}=1}^{12} \mathrm{DK} \\
& \mathrm{Em} \text { que, } \\
& \mathrm{K}^{\prime}=1,5 \log 10[(\mathrm{~T}+2,8) / \mathrm{D}]+0,5
\end{aligned}
$$

Sendo:

$\mathrm{T}=$ razão entre a demanda e o suprimento hídrico em uma região e

$\mathrm{D}=$ média mensal dos valores absolutos de $\mathrm{d}$.

A equação final do índice é:

$$
\mathrm{Xi}=(\mathrm{Zi} / 3)+0,897 * \mathrm{X}_{\mathrm{i}-1}
$$

Em que:

$\mathrm{Xi}=$ valor do índice de Palmer do mês em questão e $\mathrm{X}_{\mathrm{i}-1}=$ valor do índice de Palmer do mês precedente. 
A padronização (ponderação) de d é obtida pelo produto desse parâmetro com o fator $\mathrm{K}$, dando origem ao índice $\mathrm{Z}$ de anomalia hídrica descrito na expressão 5.

$$
\mathrm{Z}=\mathrm{d} * \mathrm{~K}
$$

A adaptação do PDSI às condições do Estado de São Paulo, teve como foco principal o fator $\mathrm{K}$ de caracterização climática. Os outros elementos da metodologia original ,como a precipitação "Climatologicamente Apropriada às Condições Existentes" e o índice d, foram calculados como descritos no trabalho original de Palmer (1965). A expressão final de K para o Estado de São Paulo é:

$\mathrm{K}=\left(22,8 \mathrm{~K}^{\prime}\right) / \Sigma \mathrm{DK}^{\prime}$

A equação final do índice adaptado para o Estado de São Paulo é:

$$
\text { PDSIadapi }=(\mathrm{Zi} / 0,94)+0,15 * \text { PDSIadapi-1 }
$$

As categorias de seca, de acordo com o PDSI adap e com o SPI são apresentadas na Tabela 1

Tendo em vista a afirmação de Santos et al., (1987) que diz que o método original de Palmer (1965) só é válido para a região na qual foi originalmente desenvolvido, esse estudo priorizou a comparação entre o PDSI adap (desenvolvido especificamente para o Estado de São Paulo) e o SPI (índice de aplicação mundial).

$\mathrm{O}$ índice $\mathrm{Z}$ de anomalia hídrica e o SPI mensal quantificam as condições de umidade de um determinado mês em uma dada região sem a influência de meses precedentes (escala mensal de análise definida). Tendo em vista tal fato, foram realizadas regressões lineares entre esses dois índices a fim de se verificar a coerência entre esses dois métodos. Foi ainda calculada a correlação entre o método de Palmer (1965) e a do SPI em diversas escalas de tempo a fim de verificar a existência de uma memória inerente ao PDSI e ao PDSI adap no Estado de São Paulo.

\section{RESULTADOS E DISCUSSÃO}

A relação entre o índice $Z$ de anomalia hídrica do método do PDSIadap e o SPI mensal é apresentada na Tabela 2, para as seis localidades base desse estudo em anos secos (1963), úmidos (1982) e para a série histórica de 1960 a 2003 de cada região.

Para realizar as regressões apresentadas na Tabela 2, os valores do SPI mensal foram inseridos no eixo das ordenadas, enquanto os valores de Z (PDSIadap) no eixo das abscissas de um plano cartesiano. Em outras palavras, pela equação de regressão, quando $\mathrm{Z}$ for igual a zero, o SPI terá o valor do intercepto da mesma. Em todos os períodos de análise e em todas as localidades, o intercepto manteve-se na categoria próximo à normal do SPI (Tabela 1), demonstrando que, quando a chuva observada for igual a $\mathbf{P}$, o SPI mensal indicará condições hídricas normais de uma dada região. Essas características aliadas aos altos valores de $\mathrm{R}^{2}$ apresentados na Tabela 2, além de indicar grande coerência entre a metodologia dos dois índices de seca, indicam também que apesar de utilizar parâmetros do solo e de temperatura, o elemento de grande importância no cálculo de $\mathrm{Z}$ e conseqüentemente do PDSI adap é a precipitação pluvial.

Os coeficientes de inclinação das retas de regressão linear (Tabela 2), permitem concluir que o índice $\mathrm{Z}$ tem um comportamento semelhante ao SPI mensal, independente do local em questão, já que os valores desse foram bastante semelhantes entre as diferentes regiões para um mesmo período de análise. Esse fato é mais um indicativo de que ambos os índices são espacialmente coerentes no Estado de São Paulo.

Por se tratar de um modelo probabilístico, os valores da distribuição gama variam entre $0 \mathrm{e} 1$, assim, por maior que seja um valor de chuva, a probabilidade cumulativa de ocorrência nunca ultrapassará a unidade. Como o Índice Padronizado de Precipitação tem como base tal distribuição, altos valores de precipitações (ainda que extremos) não são capazes de gerar valores de SPI que se desviem acentuadamente de uma série histórica.

Tabela 1 - Categorias de seca de acordo com o PDSI adap e com o SPI.

\begin{tabular}{cccc}
\hline PDSI adap & Categoria & SPI & Categoria \\
\hline 0,49 a $-0,49$ & Próxima ao Normal & 0 a $-0,99$ & Seca Incipiente \\
$-0,50$ a $-0,99$ & Seca Incipiente & $-1,00$ a $-1,49$ & Moderadamente Seco \\
$-1,00$ a $-1,99$ & Ligeiramente Seco & $-1,5$ a $-1,99$ & Severamente Seco \\
$-2,00$ a $-2,99$ & Moderadamente Seco & $<-2,00$ & Extremamente Seco \\
$-3,00$ a $-3,99$ & Muto Seco & \\
$<-3,99$ & Extremamente Seco & \\
\hline
\end{tabular}


Tabela 2 - Parâmetros estatísticos da comparação entre o índice Z de anomalia hídrica e o SPI em locais do Estado de São Paulo nos anos de 1963, 1982 e no período entre 1960 e 2003.

\begin{tabular}{lcccc}
\hline \multicolumn{1}{c}{ Local } & Ano & $\mathbf{R}^{\mathbf{2}}$ & Intercepto & Inclinação \\
\hline Campinas & 1963 & 0,91 & $-0,12$ & 0,43 \\
Campinas & 1982 & 0,91 & 0,16 & 0,30 \\
\hline Campinas & 1960 a 2003 & 0,90 & 0,06 & 0,38 \\
\hline Jaú & 1963 & 0,80 & $-0,17$ & 0,41 \\
\hline Jaú & 1982 & 0,92 & 0,18 & 0,30 \\
\hline Jaú & 1960 a 2003 & 0,88 & 0,06 & 0,38 \\
Mococa & 1963 & 0,80 & $-0,14$ & 0,4 \\
Mococa & 1982 & 0,89 & 0,20 & 029 \\
\hline Mococa & 1960 a 2003 & 0,86 & 0,09 & 0,37 \\
\hline Piracicaba & 1963 & 0,92 & 0,04 & 0,47 \\
\hline Piracicaba & 1982 & 0,90 & 0,15 & 0,28 \\
Piracicaba & 1960 a 2003 & 0,88 & 0,06 & 0,38 \\
Ribeirão Preto & 1963 & 0,86 & 0,00 & 0,45 \\
Ribeirão Preto & 1982 & 0,90 & 0,20 & 0,30 \\
\hline Ribeirão Preto & 1960 a 2003 & 0,86 & 0,09 & 0,38 \\
\hline Tatuí & 1963 & 0,85 & $-0,07$ & 0,41 \\
\hline Tatuí & 1982 & 0,91 & 0,13 & 0,30 \\
\hline Tatuí & 1960 a 2003 & 0,90 & 0,05 & 0,38 \\
\hline
\end{tabular}

Esse fato pode ser exemplificado utilizando-se a altura pluviométrica registrada em Maio de 1983 na localidade de Piracicaba $(385 \mathrm{~mm})$. Nesse mês, a distribuição gama apresentou um valor de probabilidade de ocorrência acumulada de $99,84 \%$, resultando em um SPI de 2,86. Entretanto, igualando essa precipitação à magnitude de $600 \mathrm{~mm}(80 \%$ maior do que o registrado) obteve-se uma probabilidade de ocorrência de $99,99 \%$ e um SPI de 3,86 (apenas 35\% maior do que o gerado pela chuva de $335 \mathrm{~mm}$ ).

É interessante ainda ressaltar que uma altura pluviométrica de $46 \mathrm{~mm}$ observada no mês de Maio e na localidade em questão, apresenta uma probabilidade cumulativa de ocorrência de $49 \%$, enquanto a altura de $83 \mathrm{~mm}(80 \%$ maior do que essa última) tem uma probabilidade de $74 \%$. Nota-se, com isso, que o SPI possui um valor limite máximo próximo a quatro, apresentando ainda uma variação cada vez menor para valores extremos de precipitação.

Por se tratar apenas da diferença entre $\mathbf{P}$ e a chuva observada multiplicada pelo respectivo fator $\mathrm{K}$ de caracterização climática, o índice $\mathrm{Z}$ não apresenta o comportamento descrito acima pelo Índice Padronizado de Precipitação. Tal fato pode ser exemplificado ao se estudar o mês de Maio de 1983 em Piracicaba. Nesse período foi registrado um índice $\mathrm{Z}$ de 13,41 , entretanto ao igualar a precipitação a $600 \mathrm{~mm}$, o índice $\mathrm{Z}$ resultante seria de 24,96 (86\% superior ao valor de $\mathrm{Z}$ gerado pela precipitação de $335 \mathrm{~mm}$ observado no mês). Nota-se assim um aumento da magnitude do índice $\mathrm{Z}$ bastante superior ao aumento da magnitude do SPI para uma mesma variação da altura pluviométrica. Tal característica também torna difícil o estabelecimento de um valor limite máximo para o índice Z.

Essa diferença na variabilidade dos índices explica a razão de períodos úmidos, onde valores extremos de chuva são registrados, apresentarem os mais baixos coeficientes de inclinação da reta (Tabela 2) já que alturas pluviométricas extremas diminuem o ângulo de inclinação da mesma (índices $\mathrm{Z}$ inseridos no eixo das abscissas). No caso de períodos secos, tal efeito não é sentido, já que ambos os índices apresentam limites mínimos (chuva observada igual a zero) não registrando, portanto, valores tão extremos do índice $\mathrm{Z}$.

A variação do coeficiente de determinação entre o PDSI, PDSI adap e o SPI em diferentes escalas de tempo é apresentada na Figura 1

A análise da Figura 1 demonstra a variabilidade espacial da "memória" inerente ao PDSI. Nas localidades de Jaú, Mococa e Ribeirão Preto, esse índice apresentou a maior correlação com valores do SPI semestral, enquanto nas regiões de Campinas, Piracicaba e Tatuí, tal correlação apresentou-se maior em relação 
aos SPI de dez a catorze meses de escala de tempo. Tal fato pode ser uma das causas que levaram Mckee et al. (1995) e Guttman (1998) a firmarem que nunca se sabe ao certo qual escala de tempo o PDSI considera em sua análise.

Tal variabilidade dificulta a comparação de condições hídricas de diferentes regiões, pois não se pode afirmar que um determinado valor do PDSI em um dado mês na localidade de Ribeirão Preto, por exemplo, (na qual o Índice original de Palmer apresentou uma memória semestral) represente as mesmas condições hídricas que um mesmo valor desse índice representaria, no mesmo mês, na região de Campinas (na qual o PDSI apresentou uma memória anual). Essa característica dificulta o uso do PDSI como ferramenta auxiliar à tomada de decisão na adoção de medidas estaduais de combate à seca, pois impede a comparação desse método em diferentes áreas do Estado de São Paulo. Por apresentar uma escala de tempo fixa de análise, o PDSI adap apresentou a maior correlação com o SPI na escala mensal, em todas as localidades.
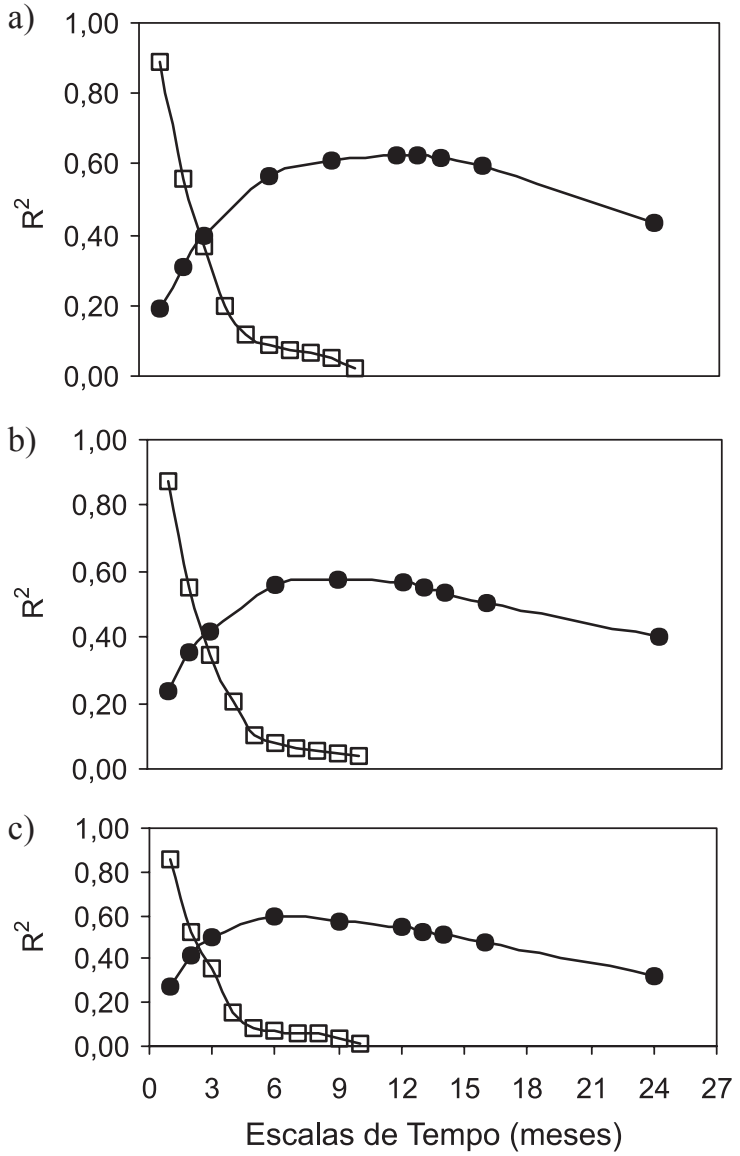

PDSI Vs SPI
A versatilidade apresentada pelo SPI, no cálculo do déficit hídrico em diversas escalas de tempo, aliada à afirmação de Hayes et al. (1999), que dizem que esse índice pode ser aplicado a qualquer região que possua uma série histórica de dados mensais de precipitação de no mínimo trinta anos, torna o Índice Padronizado de Precipitação uma valiosa ferramenta para a quantificação da seca meteorológica, hidrológica e até mesmo sócio-econômica em caráter mundial (Keyantash e Dracup, 2004). Apesar de apresentar vantagens sobre o PDSI na determinação e na definição do início/fim de uma seca, o PDSI adap ainda utiliza apenas um único valor do índice para quantificar as condições hídricas de uma região, não apresentando, portanto, a versatilidade que o SPI possui.

O primeiro passo para a correta padronização e análise do fenômeno seca, é a caracterização climática da região em estudo. Para isso, longas séries históricas de dados meteorológicos são usadas para a "calibração" regional dos índices em questão. Enquanto Karl (1986) demonstra grandes alterações na
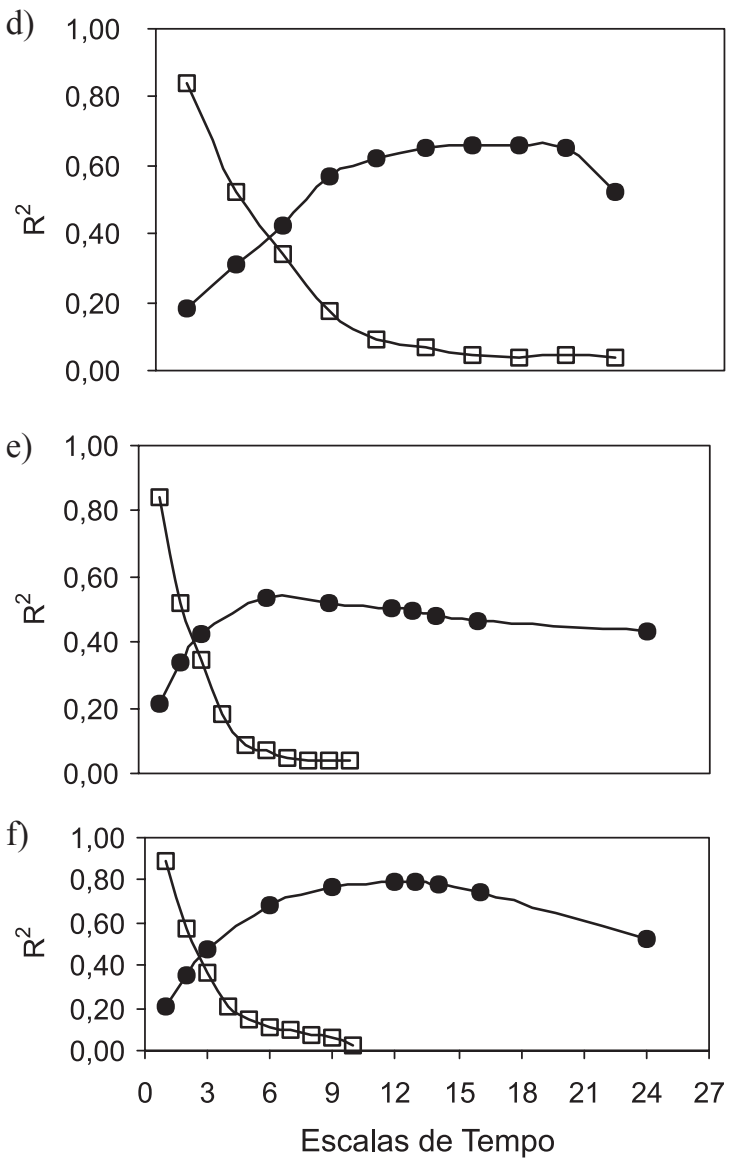

$\longrightarrow$ PDSI adap Vs SPI

Figura 1 - Variação do coeficiente de determinação entre o PDSI e SPI e entre PDSI adap e SPI em diferentes escalas de tempo para as localidades de Campinas (a), Jaú (b), Mococa (c), Piracicaba (d), Ribeirão Preto (e) e Tatuí (f). 
magnitude do PDSI em uma mesma área quando o "período de calibração" é alterado, Blain (2005) conclui que não há mudanças significativas na magnitude do SPI quando tais séries são alteradas. Essa característica aliada ao fato de utilizar apenas o elemento meteorológico mais comumente medido no Estado de São Paulo, permite que o Índice Padronizado de Precipitação possa ser aplicado a um número maior de regiões se comparado ao Índice de Palmer, que necessita, além da precipitação, outro elemento meteorológico: a temperatura do ar.

Por ser ajustado à distribuição normal, o Índice Padronizado de Precipitação, em uma longa série histórica, sempre apresentará $68 \%$ de seus valores entre 1 e $-1,95 \%$ entre 2 e -2 e $99 \%$ entre 3 e -3 . Uma interpretação relacionada é que magnitudes menores que menos um ocorram dezesseis vezes em cem anos, magnitudes menores que menos dois ocorram de duas a três vezes em cem anos e valores menores que menos três ocorram uma vez a cada duzentos anos. Com isso, notase que o SPI não é capaz de detectar regiões mais propícias à seca.

A freqüência de ocorrência de cada categoria do PDSI adap é apresentada na Figura 2.

a)

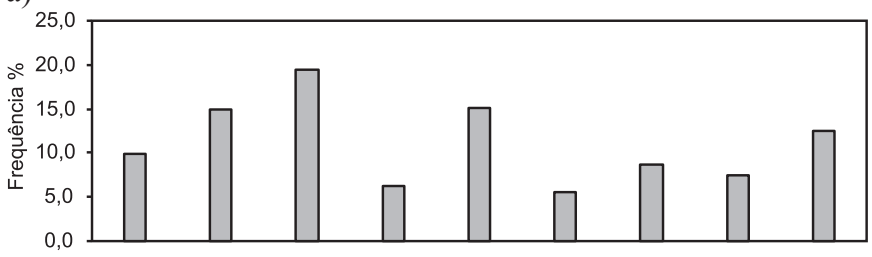

b)

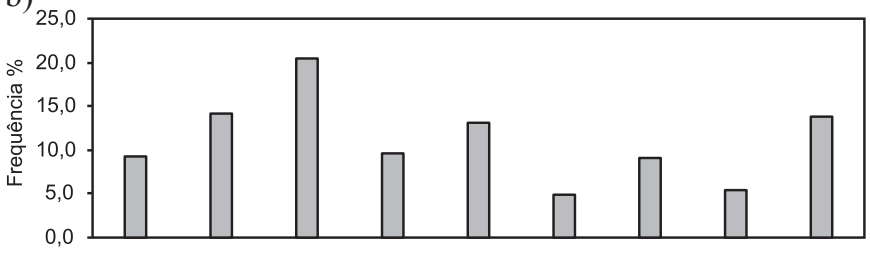

c)

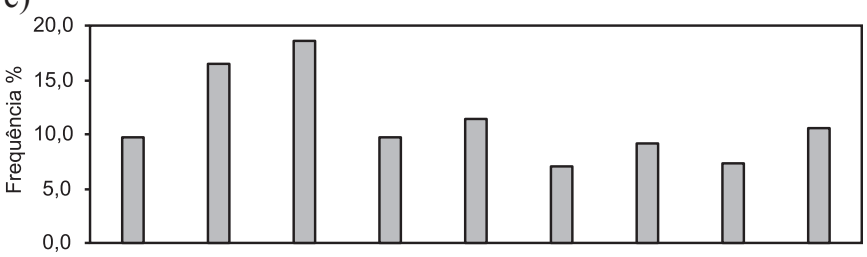

d)

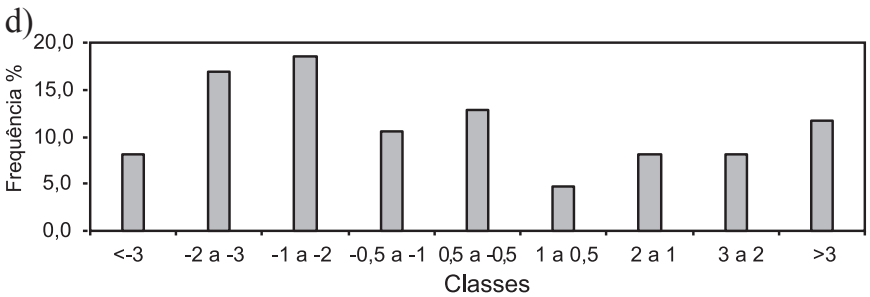

Figura 2 - Freqüência de ocorrência das classes do PDSI adap para as localidades de Campinas (a), Jaú (b), Ribeirão Preto (c) e Tatuí (d).
Como primeira análise da Figura 2 nota-se que os casos de seca extrema, apontados pelo PDSI adap, além de ocorrerem com freqüência menor do que $10 \%$ em todas as regiões do Estado, o que demonstra que o valor -3 é adequado para indicar tais eventos, apresentam ainda porcentagens de ocorrência semelhantes nas diversas localidades do Estado de São Paulo. Nota-se, também, que tal característica é conseqüencia do fato de secas extremas serem detectadas, pelo uso do Índice de Palmer adaptado, concomitantemente nas diferentes regiões do Estado.

Outra característica que pode ser notada na Figuras 2 é o fato dos eventos secos (PDSI adap menor que -0,5), ocorrerem com uma freqüência ligeiramente maior do que eventos normais ou úmidos (52,2\% secos e 47,8\% normais ou úmidos), indicando que, as diversas regiões do Estado de São Paulo, encontram-se mais freqüentemente em condições hídricas ligeiramente abaixo da normal. Tal fato é explicado por autores como Dale (1968), Cunha et al. (1997) e Blain (2005) que demonstram que o valor da média aritmética mensal de precipitação pluvial apresenta, Estado de São Paulo, uma freqüência de ocorrência inferior a $50 \%$. Assim, o uso de valores médios tanto na metodologia de Palmer (1965) como na do PDSI adap pode inserir em um requerimento relativamente exagerado de chuva para que tais índices indiquem condições hídricas úmidas em uma região.

\section{CONCLUSÃO}

O Índice Padronizado de Precipitação é uma ferramenta versátil, espacialmente consistente (invariável) e de fácil interpretação devendo ser utilizada na análise dos riscos, mitigação e implicações do fenômeno seca nos diversos segmentos da sociedade.

O método de Palmer (1965), denominado pela sigla PDSI, pode ser utilizado no Estado de São Paulo em sua forma adaptada (PDSI adap) para a quantificação da seca meteorológica na escala mensal. A comparação entre os três índices permitiu detectar que o PDSI adap possui escala temporal de análise constante (mensal) não sendo, tal característica, observada no PDSI.

\section{REFERÊNCIAS BIBLIOGRÁFICAS}

ALLEY, W.M. The Palmer drought severity index: limitations and assumptions. Journal of Climate and Applied Meteorology, Boston, v.23, 1984, p. 1100-1366.

BLAIN, G. C. Avaliação e adaptação do Índice de Severidade de Seca de Palmer (PDSI) e do Índice Padronizado de Precipitação (SPI) às condições climáticas do Estado de São Paulo. Campinas: IAC, 2005. 120f. Dissertação (Mestrado em Agricultura Tropical e Sub-tropical) - Instituto Agronômico, Campinas - SP, 2005. 
CUNHA,A.R.; MARTINS, D.; PASSOS, J.R.S. O modelo gama de probabilidade aplicado ao estudo da distribuição da chuva mensal na região administrativa de Bauru-SP. In: CONGRESSOBRASILEIRODEAGROMETEOROLOGIA 10. Anais..., Piracicaba: Sociedade Brasileira de Agrometeorologia, 1997, p. 107-109.

DALE, R. F. Applied Climatogy. West Laffayte, Indiana: Purdue University, 1968, 125p.

GUTTMAN, G.B. Comparing the Palmer Drought Index and the "Standardized Precipitation Index". Journal of the American Water Resources, Washington, v.16, n.2, p. 113-121, 1998.

HAYES, M. J.; SVOBODA, M.D.; WILHITE, D.A.; VANYARKHO, O.V, Monitoring the 1996 drought using the Standardized Precipitation Index. Bulletin of the American Meteorology Society, v. 80, n. 3, p.429-438, 1999.

KARL, T. The sensitivity of the Palmer drought severity index and Palmer's Z-index to their calibration coefficients including potencial evapotranspiration. Journal of Climate and Applied Meteorology, Boston, v.25, p. 77-86, 1986.

KEYANTASH, J. DRACUP, J.A, The quantification of drought: An evaluation of drought indices. Bulletin of the American Meteorology Society, v. 83 n.8, p.1167-1180, 2004.
MCKEE,T.B.; DOESKEN, N.J.; KLEIST,J. The relationship of drought frequency and duration to times scale. In: CONFERENCE ON APPIED CLIMATOLOGY, 8., 1993, Boston. Anais... Boston: American Meteorological Society, 1993. p.179-184. (Preprints)

MCKEE,T.B.; DOESKEN, N.J.; KLEIST,J. Drought monitoring with multiple times scales. In: CONFERENCE ON APPIED CLIMATOLOGY, 9., 1995, Boston. Proceedings... Boston: American Meteorological Society, 1995. p.233-236. (Preprints).

PALMER, W.C. Meteorological drought. Washington, 1965. 58p. (US Weather Bureau Res. $\mathrm{N}^{\circ} 45$ )

SANTOS, J. M.; MORAES, J.C.; PAIÃO, L.B.F. O índice de aridez de Palmer: uma análise crítica. In: CONGRESSO BRASILEIRO DE AGROMETEOROLOGIA. Anais..., Belém, Sociedade Brasileira de Agrometeorologia, p. 296301,1987.

THORNTHWAITE, C.W. \& MATHER, J.R. The water balance. Climatology, Centerton, v.8, n.1, p.1-40, 1955.

THOM, H. C. S. Same methods of climatological analyses. Geneva World Meteorological Organization, 1966, 53p. (WMO, 199; TP, 103; Technical note., 81.) 